\title{
A 9-year-old girl with Kawasaki disease and pulmonary nodules
}

\author{
Yousuke Higuchi $^{1}$ (D) Motoharu Ochi ${ }^{1} \cdot$ Junya Shimizu $^{1} \cdot$ Mahoko Furujo $^{1}$
}

Received: 11 May 2020 /Revised: 1 June 2020 / Accepted: 2 June 2020 / Published online: 12 June 2020

(C) International League of Associations for Rheumatology (ILAR) 2020

\section{Presentation}

A 9-year-old girl was referred for low-grade fever, posterior cervical pain, maculopapular rash, erythema of pharyngeal mucosa, and bilateral conjunctival injection on 28 January 2020. The remaining examination was unremarkable, including auscultation, meningeal irritation signs, cervical lymphadenopathy, and extremity changes. She and her family had no travel history. Laboratory data showed slightly elevated white blood cell (WBC) count $9.7 \times 10^{9} / \mathrm{L}$ and C-reactive protein (CRP) levels $3.3 \mathrm{mg} /$ L. She scheduled a follow-up visit for 3 days' time but returned later that evening complaining of chest pain. A chest radiograph showed no apparent findings, and she went home. The next day, she revisited our hospital due to a high-grade fever, neck pain, and cough. Rapid antigen tests for group A streptococcus, adenovirus, and influenza $\mathrm{A} / \mathrm{B}$ were negative. Repeated blood tests showed elevated WBC count $13.4 \times 10^{9} / \mathrm{L}$ and CRP levels $82.0 \mathrm{mg} / \mathrm{L}$; therefore, she was admitted for further examination.

Contrast-enhanced computed tomography (CT) on the second hospital day revealed no abnormalities in the neck region, but pulmonary nodules, pleural and pericardial effusion, and gallbladder hydrops were identified (Fig. 1a). Serum antibodies against Epstein-Bar virus, cytomegalovirus, and parvovirus B19 showed uninfected pattern. Blood culture and cryptococcal half quantity test

Yousuke Higuchi

yhiguchi218@gmail.com

1 Department of Pediatrics, National Hospital Organization Okayama Medical Center, 1711-1, Tamasu, Kita-ku,

Okayama, Okayama 701-1192, Japan were negative. Therefore, she was diagnosed with an incomplete Kawasaki disease (KD). We administrated intravenous immunoglobulin single-dose $2 \mathrm{~g} / \mathrm{kg}$ and aspirin $50 \mathrm{mg} / \mathrm{kg} /$ day and her symptoms and laboratory results rapidly improved. Her pericardial effusion gradually decreased, with no coronary artery abnormalities detected. On the thirteenth hospital day, follow-up CT resolved the findings (Fig. 1b), and serum mycoplasma pneumoniae particle agglutination antibody titer was not elevated.

\section{Discussion}

Pulmonary nodules are described as sub-symptom in the American Heart Association scientific statement on KD, as well as pericarditis and gallbladder hydrops [1]. There have only been seven reported cases of infants affected by pulmonary nodules associated with $\mathrm{KD}$, all of which also presented with coronary artery involvement [2-5]. Histological study of the nodules showed inflammatorycell infiltration as is seen in coronary artery aneurysms in patients with KD [2]. Rapid involution of pulmonary nodules via standard KD treatment may reflect the inflammatory nature of the lesions. We believe that the involvement of severe acute respiratory syndrome coronavirus 2 was quite low because she was brought to our 
Fig. 1 a CT scan of the chest abdomen showed pulmonary nodules with a halo sign, pleural and pericardial effusion, and acalculous gallbladder hydrops. b Twelve-day follow-up CT showed the disappearance of pulmonary nodules and fluid retention

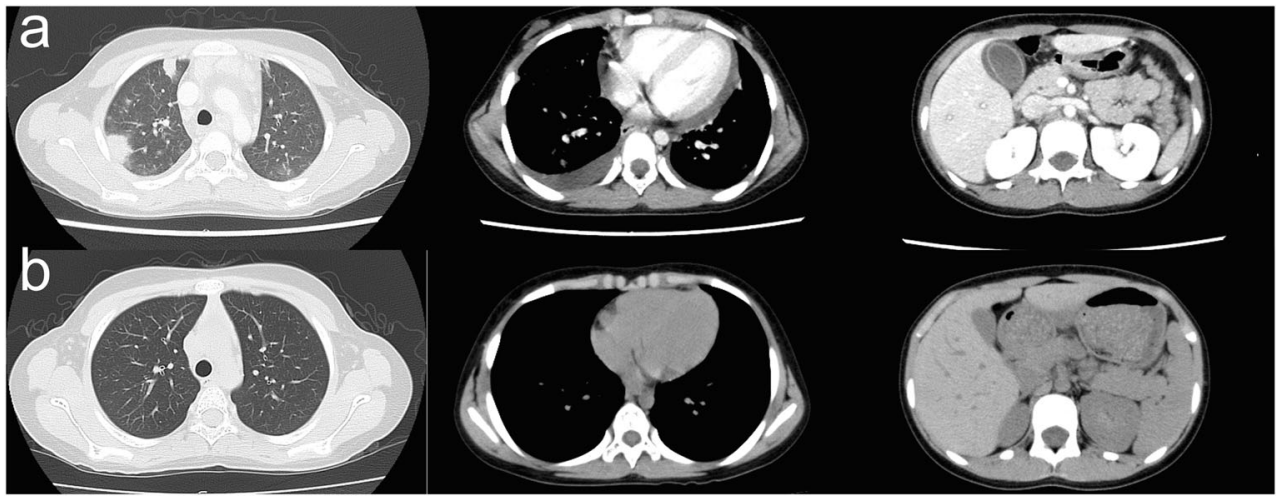

hospital in late January 2020 when the coronavirus disease epidemic was not yet in Japan. KD should also be considered as a differential diagnosis in patients presenting with acute febrile illness with pulmonary nodules, even in older children.

Acknowledgments We are grateful to the patient and her family for participating in this report. We would like to thank the radiology department at National Hospital Organization Okayama Medical Center for image interpretation. We also would like to thank Editage (www.editage.com) for English language editing.

Authors' contributions All authors contributed to the treatment, conception, and design. The first draft of the manuscript was written by Yousuke Higuchi and all authors commented on previous versions of the manuscript. All authors read and approved the final manuscript.

\section{Compliance with ethical standards}

\section{Disclosures None.}

Ethics approval All procedures performed in this study were in accordance with the ethical standard of National Hospital Organization Okayama Medical Center and with the 1964 Helsinki declaration and its later amendments. IRB approval was waived.

Consent for publication We obtained written informed consent from the patient and her parents for publication of this case report together with any accompanying images.

\section{References}

1. McCrindle BW, Rowley AH, Newburger JW et al (2017) Diagnosis, treatment, and long-term management of Kawasaki disease: a scientific statement for health professionals from the American Heart Association. Circulation 135:e927-e999. https://doi.org/10.1161/ CIR.0000000000000484

2. Freeman AF, Crawford SE, Finn LS, López-Andreu JA, FerrandoMonleón S, Pérez-Tamarit D, Cornwall ML, Shulman ST, Rowley AH (2003) Inflammatory pulmonary nodules in Kawasaki disease. Pediatr Pulmonol 36:102-106. https://doi.org/10.1002/ppul.10333

3. Monedero Picazo MD, Gómez Fernández-Montes J, Molina Fábrega R, Vallcanera Calatayud A, Alabau Vázquez G, Amparo Revert Ros M (2006) Hallazgos radiológicos pulmonares en la enfermedad de Kawasaki. Radiologia 48:14-18. https://doi.org/10.1016/S00338338(06)73124-1

4. Itani MH, Zakhour RG, Haddad MC, Arabi MT (2010) Prolonged fever with pulmonary nodules in a 4-month-old baby. Pediatr Infect Dis J 29:784. https://doi.org/10.1097/INF.0b013e3181e46ac0

5. Akagi K, Abe J, Tanaka K, Tomotaki S, Iki Y, Ueda K, Nakata M, Yoshioka T, Shiota M, Hata A, Watanabe K, Hata D (2017) Kawasaki disease with pulmonary nodules and coronary artery involvement: a report of two cases and a review of the literature. Int J Rheum Dis 20:1862-1864. https://doi.org/10.1111/1756-185X. 12692

Publisher's note Springer Nature remains neutral with regard to jurisdictional claims in published maps and institutional affiliations. 